\title{
MAGNETIC AND STRUCTURAL CHARACTERIZATION OF BIOGENIC MAGNETITE
}

\author{
M.R. McCartney* and R. E. Dunin-Borkowski** \\ *Center for Solid State Science, Arizona State University, Tempe, AZ 85287-1704. \\ **Department of Materials Science, Cambridge University, Cambridge, UK
}

Magnetic crystals below $100 \mathrm{~nm}$ in size occur in organisms in many biological phyla[1]. For example, magnetotactic bacteria contain magnetosomes, which are intracellular, ferrimagnetic crystals of magnetite $\left(\mathrm{Fe}_{3} \mathrm{O}_{4}\right)$ or greigite $\left(\mathrm{Fe}_{3} \mathrm{~S}_{4}\right)$. The magnetosomes are usually arranged in one or more linear chains within each bacterium and impart a permanent magnetic moment to the cell that results in its alignment and motion parallel to geo-magnetic field lines[2]. This behavior is thought to increase the efficiency with which such bacteria find their optimal oxygen concentrations or redox potentials at sediment-water interfaces or in water columns[3]. This fascinating phenomenon is of interest to both lay people and to scientists across many disciplines. Recently, evidence of similar magnetite crystals found in the Martian meteorite ALH84001 have been used to posit the possibility of life on Mars[4]. This identification relied on a comparison of the morphology of the meteoritic magnetite crystals with the magnetosomes from the magnetotactic bacteria, MV-1.

Clearly, what is needed is a comprehensive investigation into the morphology of biogenic magnetite. Transmission electron microscopy (TEM) bright field imaging is a projection technique and is relatively insensitive to the thickness of the sample. However, instead of obtaining brightfield images of crystals and analyzing their outlines to determine morphologies, it is possible to measure the projected thickness of a nanocrystal in the TEM in one of three ways: (i) by using energy selected imaging to form three-window, background-subtracted chemical maps [5], (ii) by using high-angle annular dark-field (HAADF) imaging with the microscope in scanning TEM mode[6]; or (iii) by using electron holography[7].

Figure 1 shows a TEM image of a chain of magnetosomes from Itaipu, Brazil. The magnetosomes are regular in size and the outline of the crystals reveals a symmetry which repeats every other crystal. Fig. 2 is a high-resolution image of a corner of one of the crystals and shows the relation between the magnetite lattice and some of the facets of the crystal. Electron holography can be used in conjunction with careful tilting of the crystals to reveal the projected thickness and thus the threedimensional morphology. Figure 3 show such an example where the electrostatic and magnetic phase shifts have been separated to show the projected thickness of several of the crystallites. Tiltiing experiments show that the crystallites are elongated along the (111) direction and have an hexagonal cross-section bounded by [110] planes. On the contrary, magnetite crystals from aquatic bacteria MS-1 have an octagonal shape bounded by [111] planes, as shown in Fig. 4. Perhaps, the best way to determine the morphology of these magnetosomes, is to use HAADF images as a series of tilt images for tomography. Figure 5 shows a processed projection of a magnetosome from a tomography series of 60 images taken at tilts of $+/-60^{\circ}$. A catalogue of 3-D images of biogenic magnetite would be an invaluable guide in evaluating extraterrestrial material for signs of life.

\section{REFERENCES}

[1] H. A. Lowenstam and S. Weiner, On Biomineralization, Oxford Univ. Press, New York, 1989.

[2] R. B. Frankel, Annu. Rev. Biophy. Bioeng. 13 (1984) 85.

[3] R. B. Frankel, et al., Biophys. J. 73 (1997) 994.

[4] K.L. Thomas-Keprta, et al., Proc. Natl. Acad. Sci. USA 98 (2001) 2164.

[5 ] R.E. Dunin-Borkowski, et al., Science 282 (1998) 1868,

[6] P.R. Buseck, et al, Proc. Natl. Acad. Sci. USA 98 (2001) 13490.

[7 ] M. R. McCartney, et al., Eur. J. Mineral. 13 (2001) 685. 


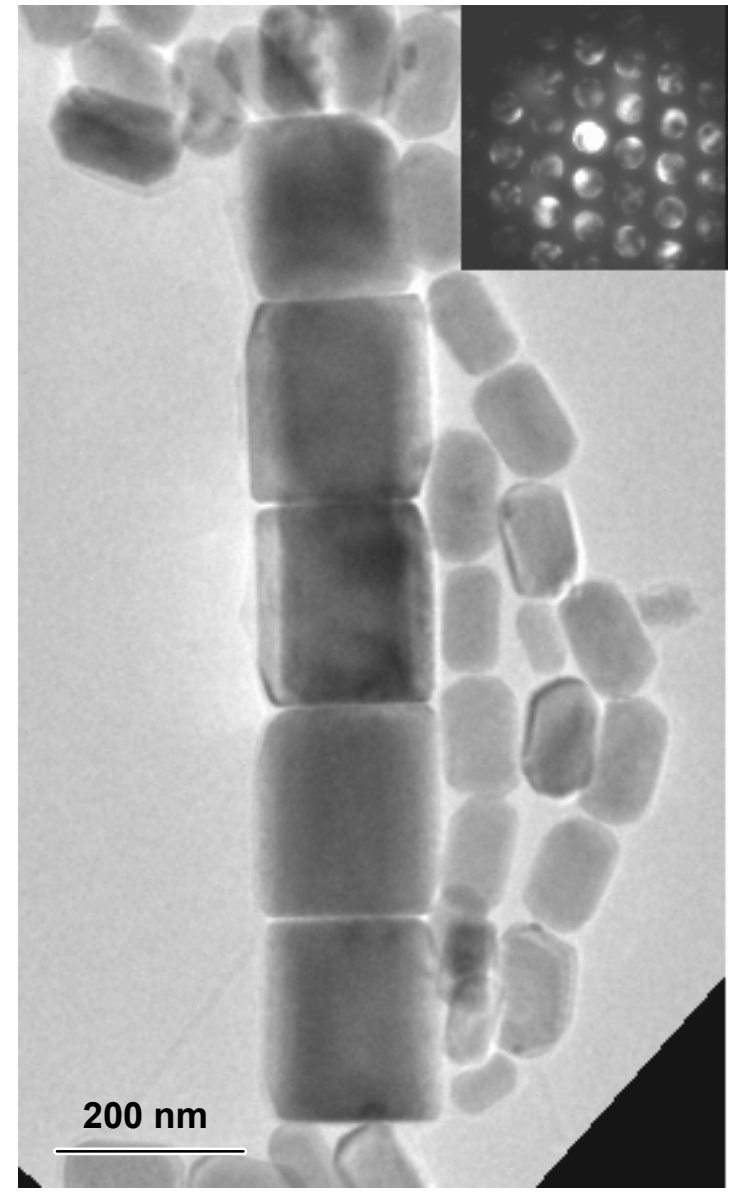

FIG. 1 TEM image of chains of magnetosomes from two different magnetotactic bacteria from Itaipu Brazil. Inset shows nano-diffraction pattern of one of the large crystals.
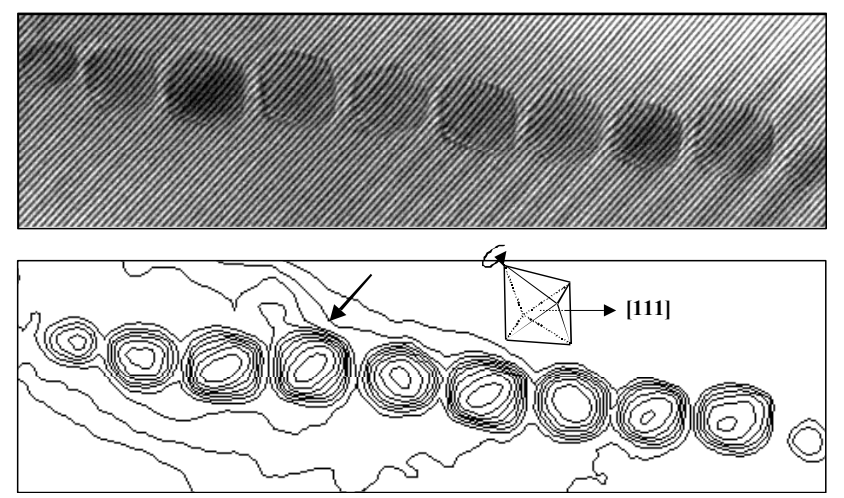

FIG. 4 Hologram and electrostatic contribution to the phase of a chain of magetite crystal from bacteria MS-1. The crystals are octahedral with (111) facets.

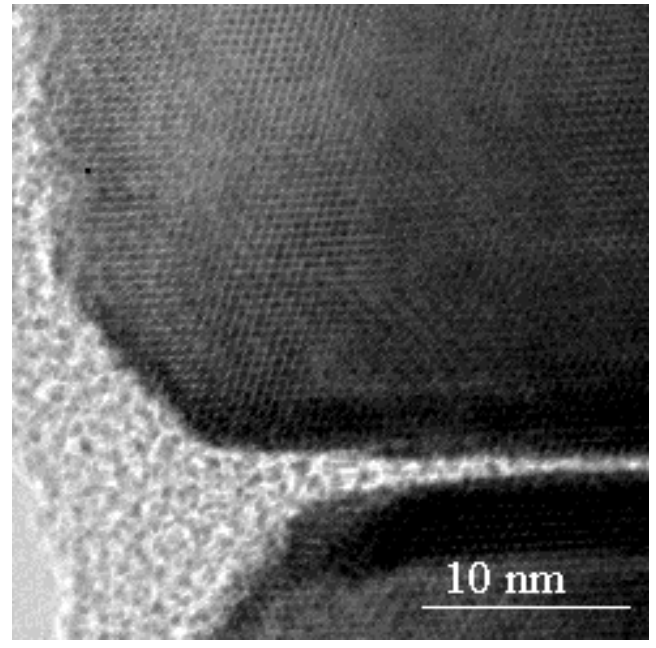

FIG. 2. High resolution image of (111) and (211) lattice planes from one of the larger crystals in Fig. 1.

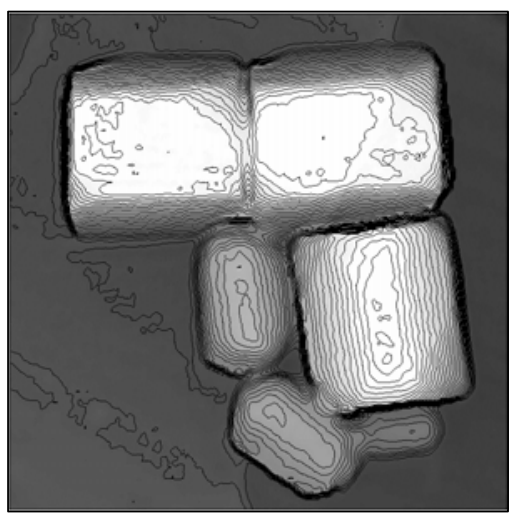

FIG. 3. Electrostatic contribution to the holographic phase shift for large Itaipu magnetosomes reveals projected thickness.

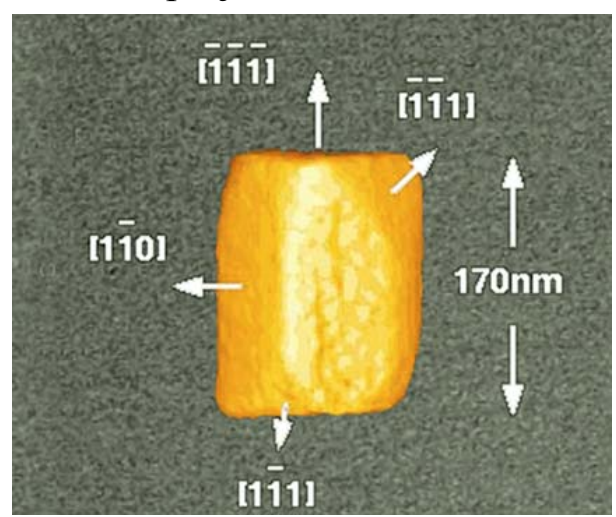

FIG. 5. Tomographic image of biogenic magnetite. 